\title{
In the PINK
}

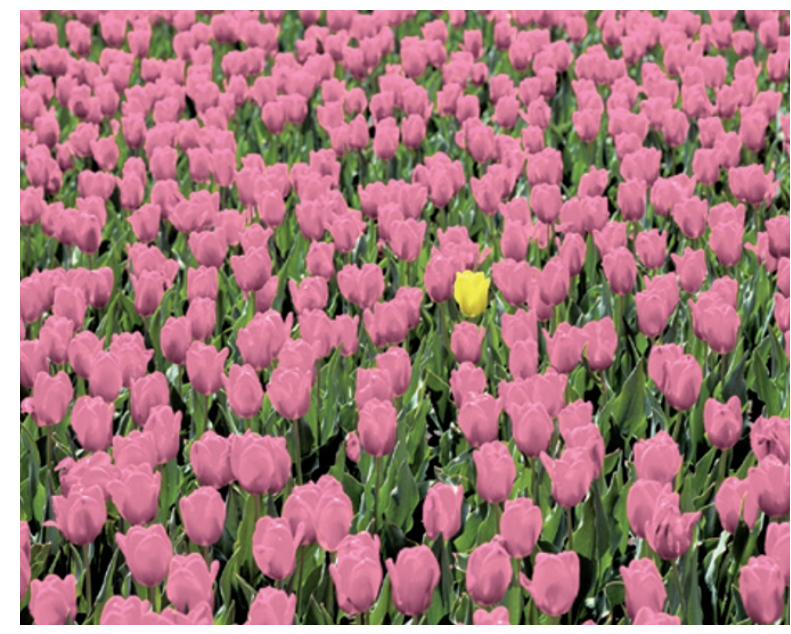

Parkinson's disease (PD) has been associated with mitochondrial dysfunction and, in some cases, with mutations in specific genes, such as parkin and PTEN-induced kinase 1 (PINK1). Flies with mutations in parkin or Pink1 show mitochondrial dysfunction, but how the genes contribute to mitochondrial integrity is unknown. Two new studies published in PNAS shed light on the mechanisms by which PINK1 and parkin influence mitochondrial function, by showing their role in fission and protection against mitochondrial toxins.

Haque et al. tested the role of PINK1 in the neuron loss that is caused by exposure to MPTP and its metabolite MPP+ (a mitochondrial toxin), both of which are used to generate animal models of PD. The authors used small interfering RNA to downregulate Pink1 expression in mouse primary neurons, and showed that this reduced neuronal survival in the presence of MPP+. Conversely, Pink1 overexpression had a protective effect. This required PINK1's kinase activity, as a mutant PINK1 in which the kinase activity was abolished was not protective. Interestingly, a PINK1 mutant lacking the putative mitochondrial targeting motif also protected neurons from MPP+ toxicity, indicating that targeting to the mitochondria is not required for the protective effect.

PINK1 had similar actions in vivo: overexpression of wild-type Pink1 in the substantia nigra protected dopamine neurons against the neurotoxic effects of MPTP injections. Again, this required PINK1's kinase activity but not the mitochondrial targeting motif, indicating that PINK1 might act in the cytoplasm.

How PINK1 protects neurons against mitochondrial toxins is unknown; however, a second study provides clues about its role in maintaining mitochondrial morphology. In this paper, Poole et al. explored whether PINK1 and parkin, which acts downstream from PINK1, have a role in mitochondrial fission and fusion in Drosophila melanogaster. Dynamic regulation of these opposing processes is required to maintain the morphological integrity of mitochondria. The authors examined the effect of manipulating $M f n 2$ and Opa1, which promote mitochondrial fusion, and Drp1, which promotes mitochondrial fission, in parkin- or Pink1-mutant flies.

Flies with mutations in parkin or Pink1 have a phenotype that is characterized by thoracic indentations and impaired climbing and flying owing to muscle degeneration. Overexpression of Drp1 in Pink1 mutants normalized this phenotype, as did loss-of-function mutations in Opa1 or deletion of Mfn2 in Pink1 and parkin mutants. Moreover, reducing the activity of Drp1 or of $M f n 2$ or Opa 1 suppressed and exacerbated, respectively, the eyestructure abnormalities that result from Pink1 overexpression.

These findings suggest that the phenotype of Pink1 and parkin mutants is caused by disturbed mitochondrial fission. Indeed, the severe defects in mitochondrial morphology in Pink1- and parkin-mutant flight muscles were normalized by increasing Drp1 gene dosage or reducing Opal gene dosage. Similarly, inactivation of both Pink1 and parkin in a D. melanogaster cell line by RNA interference caused changes in the structure of the cells' mitochondria, and these changes were reversed by inactivation of $M f n 2$ or Opal. Together, these data show that the PINK1/parkin pathway promotes mitochondrial fission.

It is unclear whether the findings of the two studies are linked - specifically, whether impaired cytoplasmic function of PINK1 might result in defects in mitochondrial fission with consequent mitochondrial dysfunction. Nevertheless, these data provide evidence for two ways in which mutations in PINK1 and parkin might lead to mitochondrial dysfunction and, possibly, PD.

Leonie Welberg

ORIGINAL RESEARCH PAPERS Poole, A. C. et al. The PINK1/Parkin pathway regulates mitochondrial morphology. Proc. Natl Acad. Sci. USA. 105, 1638-1643 (2008) | Haque, M. E. et al. Cytoplasmic Pink1 activity protects neurons from dopaminergic neurotoxin MPTP. Proc. Natl Acad. Sci. USA 105, 1716-1721 (2008) 